\title{
Variables que influyen en la elección de los estudios de Ciencias de la Comunicación en España
}

\author{
Javier SIERRA SÁNCHEZ \\ Universitat Abat Oliba CEU \\ jsierras@uao.es
}

Recibido: 4 de octubre de 2011

Aceptado: 10 de septiembre de 2012

\begin{abstract}
Resumen
En el presente trabajo de investigación empírica ahondamos en los factores y las fuentes de información que un alumno de Ciencias de la Comunicación utiliza para seleccionar la universidad y el grado. Además nos proponemos: diseccionar las características académicas, culturales y sociodemográficas de los alumnos que deciden estudiar un grado de Comunicación, averiguar cuáles son las fuentes de información que consultan para tomar la decisión, conocer cuáles son las factores de elección más valorados y por último, descubrir el papel que cumplen los padres y los amigos a la hora de decantarse por estudiar un grado de comunicación. Para conseguir estos objetivos hemos desarrollado una metodología cuantitativa basada en la elaboración de un cuestionario. Los resultados han sido contundentes, los factores de elección más importantes para los alumnos de Ciencias de la Comunicación son aquellos relacionados con la excelencia, la calidad y la reputación de la facultad/universidad. Por otro lado, hemos comprobado que las fuentes de elección mejor consideradas por estos alumnos son las que tienen una relación directa y experiencial con respecto a la Universidad y/o al grado.

Palabras clave: Comunicación, Comportamiento del consumidor, Comunicación y Educación, Contexto Universitario, Diagnóstico, Educación en Comunicación, Educación Superior, Formación, Sociología del Consumo.
\end{abstract}

\section{Factors Influencing a Student's Decision to Pursue a Communications Degree in Spain}

\begin{abstract}
This paper describes empirical research on the sources of information that future communication sciences students utilise to weigh their higher education options and the factors that influence their choices of universities and degree programmes. We begin by identifying the academic, cultural and sociodemographic characteristics of students who choose communication sciences as a university career and then move on to explore the information sources they consult to learn about universities and the programmes they offer, the factors that influence their choices, and the roles that friends and family play in their decision to pursue a degree in the communication sciences. Research data for this study was gathered using a quantitative methodology that incorporated a survey of first-year undergraduate communication sciences students throughout Spain. Results emphatically demonstrate that a student's decision where to study communication sciences is most influenced by the quality and excellence of a university's programmes and its reputation. The study also revealed that students seeking information on universities and degree programmes placed the highest value on direct and experiential sources and channels.

Keywords: Communication, consumer behaviour, communication and education, university environment, diagnostics, communication studies, higher education, training, Sociology of Consumption

Referencia normalizada: SIERRA SÁNCHEZ, Javier (2012): "Variables que influyen en la elección de los estudios de Ciencias de la Comunicación en España”. Estudios sobre el mensaje periodístico, vol. 18, núm. 2 (julio-diciembre), págs.: 925-937. Madrid, Servicio de Publicaciones de la Universidad Complutense.
\end{abstract}

Sumario: 1. Introducción. 2. Metodología. 3. Resultados: descripción y análisis. 4. Contrastación de hipótesis y conclusiones. 5. Referencias bibliográficas. 


\section{Introducción}

La Universidad española ha sufrido una gran transformación desde su adaptación al EEES (Espacio Europeo de Educación Superior). Diferentes estudios de la Comisión Europea (COM 2002) y (COM 2003), Ministerio de Educación (Estrategia Universidad 2015), Fundación Conocimiento y Desarrollo (Informe CYD 2009), Oficina de Cooperación Universitaria (2020 Tendencias Universidad: Estudio de Prospectiva) y Círculo de Empresarios (Una universidad al Servicio de la Sociedad, 2007) proponen un replanteamiento de la misión que debe cumplir la Universidad solicitando a ésta una gestión sostenible y autofinanciable basada en procesos, sistemas de garantía de calidad, competitividad y optimización de recursos. Tal y como indican (Veloutsou, LEWIS y PATON, 2004) las universidades trabajan hoy como las corporaciones y las grandes empresas para sobrevivir en un contexto marcado por: la globalización, el surgimiento de nuevas universidades, descenso de la natalidad, el aumento de la educación a distancia por el avance de las TIC y por disminución de la demanda mientras que la oferta ha aumentado y se ha diversificado.

La transferencia de las competencias en materia de educación a los gobiernos autonómicos ha otorgado a las universidades mayor autonomía de gestión. Como contrapartida, se les solicita que sean sostenibles y en la medida de lo posible, autofinanciables. De este modo, el estudiante se convierte en un factor clave para la financiación. Las universidades públicas reciben una determinada cantidad económica de financiación en función del número de estudiantes que albergan en las aulas. Para las universidades privadas, el importe de la matrícula de sus alumnos es la principal fuente de financiación. Por eso las universidades compiten por captar el mayor número de estudiantes (COMM y LABAY, 1996; LANDUM, TUNISI y HARLESS ,1998; LUQUE Y BARRIO, 2007).

De ahí que surja la necesidad de este estudio que pretende conocer cuáles son los factores de elección y las fuentes de información más importantes a la hora de que los alumnos decidan dónde y porqué estudiar Ciencias de la Comunicación en España. (Del Olmo, 2009) señala que en los países anglosajones los estudios sobre los factores de elección de Universidad se han multiplicado a partir de la implementación, entre otros, de políticas que pretenden estimular la competencia entre las instituciones a través de medidas como la financiación de la demanda, la evaluación de los resultados y el suministro de información a las familias. Estos hechos provocan que los dirigentes de las universidades se preocupen cada vez más por conocer cuáles son los factores y preferencias de los alumnos al elegir una universidad.

El contexto universitario español, marcado por una fuerte competencia como consecuencia de un aumento y diversidad de la oferta formativa e incremento de universidades, sumado al descenso paulatino de la natalidad, hace que la institución universitaria tenga que recurrir al marketing para poder captar nuevos alumnos. Los primeros trabajos sobre el marketing aplicado a universidades fueron desarrollados en Gran Bretaña y EEUU a principio de la década de 1980, destacamos (DAVIES \& SCRIBBINS, 1985; KEEN \& WARNER, 1989). Otras aportaciones posteriores muy interesantes son las de (Aliff, 1998 ; Baldwin, 1994; Delmonico, 2000; Pitman, 2000; SHUPE, 1999; TIERNEY, 1999). 
Desde esta perspectiva el factor de elección es analizado como un proceso de compra y el alumno es visto como un consumidor. Fue Chapman en 1986 el primero que aplicó el comportamiento de compra a la educación. Desde la psicología del comportamiento del consumidor, el estudiante, al seleccionar una universidad y/o grado/máster pasa por varias fases: evaluación anterior a la compra, el propio proceso de compra y evaluación de la postcompra. Desde entonces han proliferado los estudios interesados en el comportamiento del estudiante como consumidor (ATHIYAMAN ,1997; Osterbeek, Groot y Hartog, 1992; LANDrum, TurRisi y Harless, 1998; IVy, 2001; SOUtAR y Turner 2002; MARINGe, 2006; TChibOzo, 2007; Chen, 2009).

En paralelo, otros estudios se han detenido en analizar concretamente los factores de elección de universidad y grado; destacamos las aportaciones de (FIELDEN, Milton y Motes, 1993; Meringe ,2006; SoutAr y Turner, 2002, HoldSworth y Nind, 2005). Los trabajos más importantes de investigadores españoles sobre factores de elección son los de (BeErli y Díaz, 2003; Rahona, 2006); Fuentes, Gil y Moliner, 2007; Cervera y Ruiz, 2007; LuQue y Del Barrio, 2007; GonzÁlez Gallarza et al, 2008; Del Olmo, 2009).

A partir del marco teórico planteado hemos formulado tres hipótesis que trataremos de demostrar en este trabajo de investigación.

Hipótesis 1. Los factores de elección más importantes para los alumnos de Ciencias de la Comunicación son aquellos que están relacionados con la excelencia, la calidad y la reputación, en sintonía con los principios inspiradores de la Declaración de Bolonia.

Hipótesis 2: Las fuentes de información más valoradas por los alumnos de Ciencias de la comunicación son las que tienen una relación directa y experiencial con respecto a la Universidad y/o al grado.

Hipótesis 3: Los estudiantes de Ciencias de la Comunicación en España prefieren estudiar Ciencias de la Comunicación en una universidad Pública.

\section{Metodología}

Se ha realizado una extensa revisión de la literatura con la finalidad de focalizar el objetivo de la investigación y encontrar el mejor método científico para alcanzar los objetivos propuestos y dar respuestas solventes a las hipótesis formuladas. La búsqueda se ha realizado principalmente utilizando bases de datos de revistas electrónicas (ISIWeb of Knowlegde, Scopus, ABI-INFORM, ECONLIT, Publish or Persih, entre otras) así como también, en el seno de las revistas más significativas del área del sector del marketing y de educación (International Journal of Public Sector Management, Studies in Higher Education, Journal of Higher Education Policy and Management, International Journal of Educational Management, Journal of Marketing for Higher Education, Journal of Education for Business, Journal of Professional Services Marketing)

Para poder demostrar las hipótesis y alcanzar los objetivos planteados, hemos confeccionado un cuestionario telemático a través de la aplicación Limesurvey que constaba de veinticuatro preguntas cerradas en algunos casos se les instaba a que otorgasen una puntuación, en otras se les solicitaba que hicieran un escala de valoración y por 
último, se les pedía que, sobre una lista predeterminada de elementos, señalaran con una " $x$ " aquella respuesta que correspondiera.

Tabla 1. Cuadro-resumen de los parámetros de la investigación empírica

\begin{tabular}{ll}
\hline Enfoque de la investigación & Empírica, exploratoria y descriptivo-analítica \\
\hline Método & Cuantitativo (cuestionario) \\
\hline Población & Alumnos de $1^{\circ}$ curso de grado de Ciencias de la Comunicación \\
\hline Método de muestreo & Muestreo por cuotas y de conveniencia \\
\hline Fecha del trabajo de campo & Marzo 2011 \\
\hline $\begin{array}{c}\text { Tipos de Facultades /Universidades } \\
\text { objeto del estudio }\end{array}$ & $\begin{array}{c}\text { Universidades en las que se imparte algún grado de Ciencias de } \\
\text { a Comunicación (Universidad pública, universidad privada } \\
\text { laica y universidad privada religiosa) }\end{array}$ \\
\hline
\end{tabular}

Los datos recogidos en cada una de las preguntas fueron codificados e introducidos en el ordenador del Centro de Datos de la Universitat Abat Oliba CEU utilizando una programación propia. Para el tratamiento y explotación de los datos se ha empleado el programa SPSS v.18.

Se invitó a participar en el Estudio a las 46 universidades que actualmente imparten primer curso de grado de Ciencias de la Comunicación en cualquiera de sus modalidades. Finalmente participaron nueve universidades/facultades de titularidad pública y otras nueve de carácter privado.

Tratamos de buscar un equilibrio en cuanto al número de alumnos que participaban en la muestra en función de la procedencia; así participaron un total de 344 alumnos en total, siendo un $52,6 \%$ alumnos procedentes de universidades públicas y un $47,4 \%$ de universidades privadas. Sin embargo, en lo que respecta a la variable género, el Informe Anual de la Profesión Periodística (2010) ya señala la tendencia de ser carreras copadas en un mayor porcentaje por chicas que por chicos.

En cuanto a la procedencia del centro en el que han estudiado el bachillerato el $51,5 \%$ de la muestra procede de un centro público, el $32 \%$ de un centro privado /concertado religioso y el $16,6 \%$ de un centro privado/concertado no religioso.

\section{Resultados: descripción y análisis}

El 86,9\% de los alumnos que acceden a los estudios de Ciencias de la Comunicación lo hacen a través de las pruebas de acceso a la Universidad (PAU), un 7\% acceden a través de la formación profesional y destacamos el hecho de que aproximadamente el $3 \%$ son alumnos que vienen a cursar estos estudios procedente de sistemas educativos extranjeros. Las personas mayores de 25 y 45 años apenas muestran interés en cursar este tipo de carreras.

Según Informe Anual de la Profesión Periodística (2010) las notas de corte para acceder a los estudios de Ciencias de la Comunicación se sitúan alrededor del notable. Esto provoca que los alumnos, tal y como observamos en el tabla anterior, se vean abocados a realizar las dos pruebas (la general y la específica) para intentar conseguir una mayor puntuación en el selectivo para poder entrar en los grados de Comunicación. De tal manera que el alumno que inicia sus estudios en estos grados es un estudiante que ha obtenido una puntuación de notable o superior para $65 \%$ de los casos. 
Tabla 2. Resultados obtenidos en las variables indicadas

\begin{tabular}{|l|l|r|r|}
\hline \multicolumn{1}{|c|}{ Variable } & \multicolumn{1}{|c|}{ Subvariable } & Núm. & \multicolumn{1}{c|}{$\%$} \\
\hline \multirow{4}{*}{$\begin{array}{l}\text { Vía de acceso a la } \\
\text { Universidad }\end{array}$} & Pruebas de acceso a la Universidad (PAU) & 299 & 86,9 \\
\cline { 2 - 4 } & Formación profesional & 24 & 7,0 \\
\cline { 2 - 4 } & Persona con titulación universitaria & 3 & 0,9 \\
\cline { 2 - 4 } & Acceso a la Universidad personas mayores de 25 años & 4 & 1,2 \\
\cline { 2 - 4 } & Acceso a la Universidad personas mayores de 45 años & 1 & 0,3 \\
\cline { 2 - 4 } & Procedente de un sistema educativo extranjero & 10 & 2,9 \\
\cline { 2 - 4 } & Convalidación parcial de estudios extranjeros & 3 & 0,9 \\
\hline \multirow{3}{*}{$\begin{array}{l}\text { Examen PAU } \\
\text { Prueba de acceso a }\end{array}$} & Han realizado solo la prueba general & 70 & 20,3 \\
\cline { 2 - 4 } la Universidad) & Han realizado la fase general y la específica & 229 & 66,6 \\
\cline { 2 - 4 } & Otros casos & 45 & 13,1 \\
\hline \multirow{2}{*}{$\begin{array}{l}\text { Notas PAU fase } \\
\text { general }\end{array}$} & De 5 a 6 & 166 & 48,3 \\
\cline { 2 - 4 } & De 6,1 a 8 & 57 & 16,6 \\
\cline { 2 - 4 } & De 8,1 a 10 & 16 \\
\hline
\end{tabular}

Para contrastar si la nota media de selectividad es diferente por colegio de procedencia (público, privado) se realiza un intervalo de confianza para la diferencia de notas medias. En los resultados que se ofrecen más abajo, tras realizar la prueba de Levene para la igualdad de varianzas, se concluye que estas son iguales, por lo que el intervalo de confianza obtenido al 95\% de confianza para la diferencia de medias es positivo, indicando que la nota media de selectividad para los que proceden de colegios públicos es superior a la nota media de los que proceden de colegios privados (independientemente de su identidad religiosa).

Tabla 3. Prueba Levene para igualdad de varianzas con el fin de conocer si existen diferencias significativas entre las nota PAU y la procedencia del centro en el bachillerato.

\begin{tabular}{|c|c|c|c|c|c|c|c|c|c|c|c|}
\hline \multicolumn{12}{|c|}{ Estadísticos de grupo } \\
\hline \multicolumn{5}{|c|}{$\begin{array}{l}\text { Tipo de centro, instituto o colegio donde has } \\
\text { cursado los últimos estudios antes de acceder a } \\
\text { tu Universidad }\end{array}$} & $\mathrm{N}$ & \multicolumn{2}{|l|}{ Media } & \multicolumn{2}{|c|}{ Desviación típ. } & \multicolumn{2}{|c|}{ Error típ. de la media } \\
\hline \multirow[b]{2}{*}{ cod_nota_pau } & \multicolumn{4}{|c|}{ Colegio público } & 160 & \multicolumn{2}{|c|}{7,2109} & \multicolumn{2}{|r|}{1,22845} & \multicolumn{2}{|r|}{, 09712} \\
\hline & \multicolumn{4}{|c|}{$\begin{array}{l}\text { Colegio privado/concertado } \\
\text { religioso }\end{array}$} & 100 & \multicolumn{2}{|c|}{6,8650} & \multicolumn{2}{|r|}{1,12210} & \multicolumn{2}{|r|}{, 11221} \\
\hline \multicolumn{12}{|c|}{ Prueba de muestras independientes } \\
\hline \multicolumn{12}{|c|}{\begin{tabular}{c|c} 
Prueba de & \\
Levene para & Prueba T para \\
la igualdad & \\
de varianzas &
\end{tabular}} \\
\hline & & \multirow[t]{2}{*}{$\mathrm{F}$} & \multirow[t]{2}{*}{ Sig. } & \multirow[t]{2}{*}{$\mathrm{t}$} & \multirow[t]{2}{*}{$\mathrm{gl}$} & \multirow[t]{2}{*}{$\begin{array}{c}\text { Sig. } \\
\text { (bilateral) }\end{array}$} & \multirow{2}{*}{\multicolumn{2}{|c|}{$\begin{array}{l}\text { Diferencia } \\
\text { de medias }\end{array}$}} & \multirow[t]{2}{*}{$\begin{array}{l}\text { Error típ. } \\
\quad \text { de la } \\
\text { diferencia }\end{array}$} & \multicolumn{2}{|c|}{$\begin{array}{l}95 \% \text { Intervalo de } \\
\text { confianza para la } \\
\text { diferencia }\end{array}$} \\
\hline & & & & & & & & & & Inferior & Superior \\
\hline \multirow{2}{*}{$\begin{array}{l}\text { cod_nota } \\
\text { pau }\end{array}$} & $\begin{array}{l}\text { asumido } \\
\text { as iguales }\end{array}$ & ,989 &, 321 & 2,283 & 258 & 023 & & ,34594 &, 15154 &, 04753 & ,64435 \\
\hline & $\begin{array}{l}\text { Ian } \\
\text { o } \\
\text { as iguales }\end{array}$ & & & 2,331 & 224,450 &, 021 & & ,34594 &, 14840 &, 05350 & ,63838 \\
\hline
\end{tabular}


En cuanto a la preferencia por estudiar en un centro, en función de la titularidad del mismo, predomina la tendencia marcada de la Universidad/facultad pública en un $69,8 \%$ mientras que un $30,2 \%$ prefieren estudiar en una Universidad/facultad privada.

En la siguiente tabla mostramos las fuentes de información mejor valoradas por la muestra objeto de estudio a la hora de seleccionar un grado y una universidad. Establecimos un ranking de las fuentes de información empleadas por los alumnos analizar cuáles son las más valoradas.

Tabla 4. Ranking de las fuentes de información empleadas por los alumnos de primer curso de los grados de Ciencias de la Comunicación de las facultades de Comunicación españolas.

\begin{tabular}{|c|c|c|c|c|c|c|}
\hline Fuentes de información & 照 & 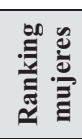 & 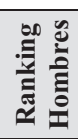 &  & 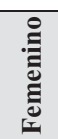 & 造 \\
\hline Página web de las universidades & 1 & 1 & 1 & 99 & 222 & 321 \\
\hline Otras publicaciones (Guías, folletos) & 2 & 2 & 4 & 65 & 170 & 235 \\
\hline Familia & 3 & 3 & 3 & 67 & 158 & 225 \\
\hline Estudiantes de la universidad & 4 & 4 & 2 & 68 & 149 & 217 \\
\hline Amigos de la escuela & 5 & 5 & 5 & 63 & 139 & 202 \\
\hline Visita personal a la universidad & 6 & 6 & 7 & 60 & 137 & 197 \\
\hline Otros amigos & 7 & 7 & 6 & 62 & 130 & 192 \\
\hline Amigos de la familia & 8 & 8 & 8 & 55 & 122 & 177 \\
\hline Visita a ferias de educación para estudiantes & 9 & 9 & 13 & 40 & 121 & 161 \\
\hline $\begin{array}{l}\text { Información proporcionada por el } \\
\text { profesor/tutor/orientador de la escuela }\end{array}$ & 10 & 10 & 9 & 49 & 111 & 160 \\
\hline $\begin{array}{l}\text { Presentaciones de la universidad en el centro escolar o } \\
\text { instituto }\end{array}$ & 11 & 11 & 12 & 44 & 108 & 152 \\
\hline Antiguos alumnos de la universidad & 12 & 12 & 10 & 47 & 96 & 143 \\
\hline Otras páginas web & 13 & 14 & 11 & 45 & 86 & 131 \\
\hline Redes sociales & 14 & 13 & 14 & 37 & 87 & 124 \\
\hline Anuncios en otros medios & 15 & 16 & 15 & 31 & 79 & 110 \\
\hline Jornadas de puertas abiertas de la universidad & 16 & 15 & 17 & 28 & 82 & 110 \\
\hline Material audiovisual (Vídeo institucional...) & 17 & 17 & 16 & 30 & 69 & 99 \\
\hline Centros de Información de la Administración & 18 & 18 & 19 & 20 & 53 & 73 \\
\hline Anuncios en prensa & 19 & 19 & 18 & 23 & 32 & 55 \\
\hline Anuncios en TV & 20 & 20 & 20 & 8 & 20 & 28 \\
\hline Anuncios en radio & 21 & 21 & 21 & 8 & 19 & 27 \\
\hline
\end{tabular}

Diferentes informes profesionales (GECA, SOFRES, Barlovento) que miden el consumo de los medios por parte de los jóvenes, han constatado la preferencia de éstos por Internet en detrimento de medios más tradicionales como la radio, el cine, la prensa o la televisión. Por eso los futuros alumnos, emplean Internet (la web institucional de la universidad) como un medio estratégico clave para obtener información que les sea de utilidad para poder realizar una adecuada elección de Universidad y de grado que quieren estudiar.

En segundo lugar, la fuente mayor empleada es la referente a folletos, catálogos de la Universidades/facultades/grados que reciben los alumnos cuando se desplazan a la propia universidad para hacer una valoración más objetiva y precisa del campus. A veces esta documentación se les entrega cuando asisten a las ferias de educación existentes en España. Para los alumnos de Ciencias de la Comunicación. Otra fuente de 
información que valoran considerablemente son aquellas referencias que sobre la Universidad o del grado proceden de la familia o de alumnos que están estudiando un grado de comunicación en una determinada universidad, y por último, las referencias de los amigos del instituto.

Respecto a las fuentes de información utilizadas para la elección de la Universidad y/o Grado, entendíamos que no tenían que ser diferentes por género, realizando un ranking de valoración de las mismas por género, hemos encontrado que su coeficiente de correlación rho de Spearman es alto y positivo, confirmando nuestra idea inicial de concordancia entre los órdenes de uso de dichas fuentes de información.

Otro objetivo que pretendíamos conseguir era conocer cuáles son los atributos (de una lista cerrada predeterminada) a los que más importancia otorgan a la hora de seleccionar una universidad o estudio de grado.

Tabla 5. Factores de elección a la hora de escoger una universidad

\begin{tabular}{|c|c|c|c|}
\hline $\begin{array}{l}\text { Factores de elección } \\
\text { Puntúa la influencia de cada uno de los siguientes factores a la hora } \\
\text { de escoger universidad, siendo } 1 \text { el valor más bajo (ninguna } \\
\text { influencia) y } 5 \text { el valor más alto (mucha influencia) }\end{array}$ & Media & Desv. típ. & Ranking \\
\hline Calidad de la enseñanza & 4,01 & 1,027 & 1 \\
\hline Reputación de la universidad & 3,83 & 1,093 & 2 \\
\hline Orientación práctica de los estudios & 3,61 & 1,087 & 3 \\
\hline Proyección internacional de la universidad & 3,57 & 1,148 & 4 \\
\hline Variedad de titulaciones oficiales ofertadas & 3,52 & 1,25 & 5 \\
\hline Nivel de innovación tecnológica en la universidad & 3,51 & 1,222 & 6 \\
\hline El plan de estudios era atractivo & 3,49 & 1,159 & 7 \\
\hline Facilidad de acceso a la universidad (pruebas, nota necesaria) & 3,44 & 1,239 & 8 \\
\hline Atractivo de las instalaciones & 3,35 & 1,264 & 9 \\
\hline Facilidad de acceso en transporte público & 3,28 & 1,338 & 10 \\
\hline Atractivo de las actividades académicas ofertadas & 3,25 & 1,08 & 11 \\
\hline La web de la Universidad & 3,2 & 1,206 & 12 \\
\hline Coste anual de los estudios & 3,15 & 1,375 & 13 \\
\hline Amabilidad en el trato del personal de la universidad & 3,15 & 1,181 & 14 \\
\hline Orientación humanista/integral de la formación & 3,12 & 1,095 & 15 \\
\hline Eficacia de la bolsa de trabajo & 3,07 & 1,236 & 16 \\
\hline Número de alumnos por clase & 3,02 & 1,278 & 17 \\
\hline Recomendación de estudiantes universitarios & 2,99 & 1,277 & 18 \\
\hline Calidad en los servicios de asesoramiento y atención al alumnado & 2,98 & 1,148 & 19 \\
\hline Reputación investigadora del profesorado & 2,93 & 1,122 & 20 \\
\hline Proximidad de la universidad al domicilio habitual & 2,93 & 1,526 & 21 \\
\hline La universidad más próxima no imparte la titulación deseada & 2,88 & 1,584 & 22 \\
\hline Recomendación de amigos & 2,88 & 1,203 & 23 \\
\hline Recomendación familiar & 2,85 & 1,255 & 24 \\
\hline Atención personalizada y seguimiento tutorial del alumno & 2,85 & 1,185 & 25 \\
\hline Docencia y otras actividades en lengua inglesa & 2,79 & 1,222 & 26 \\
\hline $\begin{array}{l}\text { [Promoción e información directa a los alumnos potenciales (visitas } \\
\text { a escuelas, mailings...) }\end{array}$ & 2,62 & 1,135 & 27 \\
\hline Posibilidad de vivir fuera del domicilio habitual & 2,59 & 1,465 & 28 \\
\hline Difusión de la universidad en los medios de comunicación & 2,55 & 1,199 & 29 \\
\hline Dificultad para acceder a una universidad pública & 2,54 & 1,518 & 30 \\
\hline Atractivo de la oferta deportiva & 2,38 & 1,231 & 31 \\
\hline Dificultad para acceder a una universidad privada & 2,21 & 1,394 & 32 \\
\hline
\end{tabular}


Fundamentalmente van orientados en tres direcciones como podemos observar en la tabla anterior: calidad y reputación de la enseñanza y de la Universidad, la "practicidad de los estudios" en dos sentidos: a) en cuanto al peso específico de las metodologías activas de aprendizaje basado en la práctica sobre el total del plan de estudios, y b) la posibilidad de encontrar empleo al terminar el grado en el mercado laboral; y por último, el factor que tiene que ver con la internacionalización de los estudios.

De nuevo, y como ya hicimos con las fuentes de información, queríamos conocer si existía concordancia ( $\mathrm{y}$ en qué grado) por género en cuanto a la valoración de los atributos que ellos consideran más importantes a la hora de decidirse por una universidad y por un grado. Para ello aplicamos la Rho de Spearman y a la luz de los datos obtenidos, podemos afirmar que existe una gran concordancia entre los resultados de las respuestas dadas por las chicas como por los chicos; es decir, existe una gran similitud en la valoración (ranking) que han otorgado a cada uno de los factores de elección.

Vemos en la siguiente tabla que los alumnos que se declinan por estudiar grados de Ciencias de la Comunicación lo deciden, mayoritariamente, ellos solos en un 65,4\% de los casos y en un 21,2\% lo deciden ellos mismos consultándolo con sus progenitores. Por lo que podemos afirmar que los alumnos de Ciencias de la Comunicación prefieren asumir ellos mismos el protagonismo en la etapa crucial de la elección de los estudios y de la Universidad.

Tabla 6. Toma de decisión de la Universidad y de los estudios

\begin{tabular}{|c|c|c|c|}
\hline Variable & Subvariable & Núm. & $\%$ \\
\hline \multirow{6}{*}{$\begin{array}{l}\text { Importancia } \\
\text { en la decisión } \\
\text { de estudiar un } \\
\text { grado de } \\
\text { Ciencias de la } \\
\text { Comunicación }\end{array}$} & Lo he decido yo sólo & 225 & 65,4 \\
\hline & $\begin{array}{l}\text { Lo he decidido yo } \\
\text { consultando a mis padres }\end{array}$ & 73 & 21,2 \\
\hline & $\begin{array}{l}\text { Lo hemos decidido juntos mis } \\
\text { padres y yo }\end{array}$ & 30 & 8,7 \\
\hline & $\begin{array}{l}\text { Lo han decidido mis padres } \\
\text { consultándomelo }\end{array}$ & 2 & 0,6 \\
\hline & Lo han decidido mis padres & 1 & 0,3 \\
\hline & No sabe/no contesta & 4 & 1,2 \\
\hline
\end{tabular}

\section{Contrastación de hipótesis y conclusiones}

En lo referente a la hipótesis primera donde afirmábamos que los factores de elección más importantes para los alumnos de Ciencias de la Comunicación son aquellos que están relacionados con la excelencia, la calidad y la reputación, en sintonía con los principios inspiradores de la Declaración de Bolonia. Por lo que hemos podido comprobar que se cumple totalmente la hipótesis al ser esos factores los que más importancia tienen en el proceso de elección. Coincidimos con los trabajos de (SERVIER, 1993; QURESHI, 1995; Lin, 1997; JAMES et al, 1999; MARTIN \& DiXON, 1991) que identifican la calidad como factor clave para la elección.

Por otro lado en la hipótesis segunda indicábamos que las fuentes de información más valoradas por los alumnos de Ciencias de la comunicación son las que tienen una relación directa y experiencial con respecto a la Universidad y/o al grado. En este sentido, de nuevo se cumple la hipótesis al comprobar que las fuentes más valoradas son aquellas en las que el alumno tiene una experiencia virtual (a través de la página web 
de la universidad, de las redes sociales) y real (visita en persona a la universidad donde le entregan folletos, cd's, regalos institucionales). Además hemos podido descubrir la importancia que tienen para ellos la familia y los amigos (de la escuela y los que ya tienen estudiando en la universidad).

En lo referido a la hipótesis tercera donde señalábamos que los estudiantes de Ciencias de la Comunicación en España prefieren estudiar Ciencias de la Comunicación en una universidad Pública, hemos podido demostrar empíricamente que esto es una tendencia real.

Otras conclusiones derivadas del estudio son:

1. Podemos afirmar que existe un marketing específicamente universitario cuyo fin es la captación y fidelización de los futuros alumnos para que se conviertan en un futuro en prescriptores de la Universidad/Facultad y del grado que hayan elegido.

2. Los alumnos que estudian Ciencias de la Comunicación en las Facultades españolas cuya procedencia sea un instituto o centro público obtienen mejores calificaciones en selectividad que los que proceden de centros o institutos privados.

En este estudio ha quedado constatado cómo la reputación, la excelencia y la calidad percibida son factores determinantes para seleccionar una Universidad. De ahí que los departamentos de Comunicación y Marketing de las Universidades (públicas y privadas) se esfuercen por proyectar esos atributos en todas las acciones de comunicación que desarrollan. Será interesante profundizar, en futuros trabajos de investigación, cómo las Universidades proyectan esos intangibles en los diferentes soportes comunicativos y cuáles son los aspectos tangibles en los que las Universidades se apoyan para conseguir posicionar esos intangibles en los futuros alumnos. Otro aspecto de interés, para futuras aproximaciones al objeto de estudio sería determinar en qué medida el coste de la matrícula repercute en la elección de una Universidad de titularidad pública o privada.

\section{Referencias bibliográficas}

ALIFF, John V. (1998): “Are students 'customers' of collegiate education?". Proceedings of the Annual General Meeting of the Georgia Academy of Science, 25 April, 1-10.

ASOCIACIÓN DE LA PRENSA DE MADRID [APM] (2010): Informe Anual de la Profesión Periodística. Madrid, APM.

ATHIYAMAN, Adee (1997): "Linking student satisfaction and service quality perceptions: the case of university education". European Journal of Marketing, vol. $31,7 / 8,528-541$.

BALDWIN, Gabrielle (1994): "The student as customer: the discourse of 'quality' in higher education". Journal of Tertiary Education Administration, vol. 16 ,1, 12533. 
BEERLI, Asunción y DÍAZ MENESES, Gonzalo (2003): "Los efectos de la imagen percibida de la universidad en la satisfacción de los estudiantes". Revista Española de Investigación de Marketing, ESIC, 7 (1), 7-25.

CERVERA, Amparo Y RUIZ, María Eugenia (2007): "Tourism Education at the University of Valencia: A Strategic Analysis". Advances in Tourism Marketing Conference. (Paper)

CHAPMAN, R. (1986): Towards a theory of college selection: a model of college serch and choice behaviour. Advances in Consumer Research, vol.13, Association for Consumer Research, Provo, Utah.

CHEN, Wei wei (2009): "Hospitality and tourism management education: an analysis of Chinese undergraduate students' motives and institution choice criteria". Assumption University of Thailand: Tourism Management, Graduate School of Business. Thesis MBA, en doc. pdf.: http://gsbejournal.au.edu/2V/Journal/HOSPITALITY\%20AND\%20TOURISM\%20MANA GEMENT\%20EDUCATION\%20AN\%20ANALYSIS\%20OF\%20CHINESE\%20UNDERGRADUATE $\% 20$ STUDENTS\%20\%20MOTIVES\%20AND\%20INSTITUTION\%20CHOICE $\%$ 20CRITERIA.pdf

CHRISTOPHER, Martin; PAYNE, Adrian; BALLANTYNE, David (1991): Relationship Marketing. Oxford, Butterworth-Heinemann.

CIRCULO DE EMPRESARIOS (2007): "Una universidad al servicio de la Sociedad". (http://www.circulodeempresarios.org/wp-content/uploads/2009/06/11.pdf) (01-02-2009)

COM (2002) 629 final (2002): "Puntos de referencia europeos en educación y formación: seguimiento del Consejo Europeo de Lisboa". Comisión de Las Comunidades Europeas, Bruselas 20-11-02.

COM (2003) 58 final (2002): "El papel de las universidades en la Europa del conocimiento. Comisión de las Comunidades Europeas", Bruselas 05-02-03.

COMM, Clare L. \& LABAY, Duncan G. (1996): "Repositioning colleges using changing student quality perceptions: An exploratory analysis". Journal of Marketing for Higher Education, Vol.7,4, 21-35.

CONSEJO DE LA UNIÓN EUROPEA (2001): “Informe del Consejo «Educación» al Consejo Europeo sobre los futuros objetivos precisos de los sistemas de educación y formación". N. ${ }^{\circ}$ doc. prec.: 5680/01 EDUC 18, Bruselas.

DAVIES, Peter \& SCRIBBINS, Keith (1985): "Marketing Further and Higher Education". York: Longman Group Ltd.

DECLARACIÓN de Bolonia (1999): "Declaración de los Ministros europeos de educación". UE. (http://europe.eu.int/comm/education/policies/educ/bologna/bologna.pdf) (10-02-2010)

DEL OLMO ARRIAGA, Josep Lluís (2009): Els factors d'elecció d'Universitat: el cas de la demanda a Catalunya. El cas de la demanda a Catalunya. [Tesis Doctoral]. Barcelona, Universitat Abat Oliba. 
DEL OLMO ARRIAGA, Josep Lluís (2009): La elección de Universidad: factores de decisión y canales de información. Madrid, Editorial CEU Ediciones.

DELMONICO, Matthew. J. (2000): "Is treating students as customers the right move for community colleges?". St Petersburg Junior College, 1-16.

FIELDEN, John S.; HILTON, Chadwick B.; \& MOTES, William.H. (1993): "Educational services marketing: a proposed system for enhanced recruitment of students". Journal of Professional Services Marketing, vol. 8, 191-205.

FONDEVILA GASCÓN, Joan Francesc; DEL OLMO ARRIAGA, Josep Lluís; \& SIERRA SÁNCHEZ, Javier (2011): "Universidades privadas, ¿un subsistema diferente?: cómo desarrollan los estudiantes sus preferencias hacia la universidad". On Line: Vivat Academia. 114. (http://www.ucm.es/info/vivataca/numeros/n114 /DATOSS.htm) (05-06-2011)

FUENTES BLASCO, María; GIL SAURA, Irene; \& MOLINER VELÁSQUEZ, Beatriz (2007): "La utilidad de las expectativas como base de segmentación predictiva de la calidad del servicio universitario". VI Congreso Internacional de Marketing Público y No lucrativo, Braga 14-15 de Junio de 2007.

FUNDACIÓN CYD [Conocimiento Y Desarrollo] (2009): "Informe de la Fundación Conocimiento y Desarrollo 2009". (http://www.fundacioncyd.org/wps/portal) (0102-2011)

GONZÁLEZ GALLARZA, Martina; FAYOS, Teresa; SERVERA, David; \& ARTEAGA, Francisco (2008): "Análisis y evaluación del servicio de formación universitaria: implicaciones para el marketing estratégico de las universidades". Revista de Investigación en Educación, $\mathrm{N}^{\mathrm{o}} 9$ (2), pp. 133-152. http://webs.uvigo.es/reined/

GRÖNROOS, Christian (1994): "From marketing mix to relationship marketing: Towards a paradigm shift in Marketing". Management Decisions, 32(2), 4-20.

GRÖNROOS, Christian (2000): Service Management and Marketing: A Customer Relationship Management Approach. New York, NY:John Wiley \& Sons.

HELGESEN, Øyvind (2008): "Marketing for higher education: A relationship marketing approach". Journal of Marketing for Higher Education, 18 (1), 50-78.

HOLDSWORTH, D.W. \& NIND, D. (2005): “Choice Modelling New Zealand High School Seniors' Preferences for University Education". Journal of Marketing for Higher Education, Vol.15 No.2, 81-104.

IVY, Jonathan (2001): "Higher education institution image: a correspondence analysis approach". International Journal of Educational Management, Vol. 15, 6, 276282.

JAMES, Richard; BALDWIN, Gabrielle; \& McINNIS, Craig (1999): "Which university? The factors influencing choices of prospective undergraduates. Evaluation and Investigations Programme". Australia, Higher Education Division. http://www.dest.gov.au/archive/highered/eippubs/99-3/whichuni.pdf 
KEEN, Clive \& WARNER, Daid (1989): Visual \& Corporate Identity: A study of identity programmes in the college, polytechnic and university environment. Leeds, Heist Publications

KEITH, Robert J. (1960): “The marketing revolution”. Journal of Marketing, vol. 24, 35-48.

LANDRUM, R. Eric \& TURRISI, Rob \& HARLESS, Clayton (1998): "University image: the benefits of assessment and modelling". Journal of Marketing for Higher Education, Vol.9, 1, 53-68.

LUQUE, Teodoro \& DEL BARRIO-GARCÍA, Salvador (2007): “Análisis del valor de las percepciones de los clientes en el diagnóstico estratégico de la universidad". Ponencia presentada en el International Congress Marketing Trends, 26-27 de enero de 2007.

MARINGE, Felix (2006): "University \& Course Choice: Implications for positioning, recruitment and marketing". International Journal of Educational Management, Vol. 20, 6, 466-479.

MARTIN, Nancy K. \& DIXON, Paul N. (1991): "Factors influencing students' college Choice". Journal of College Student Development, 32(3), 253-257.

MINISTERIO DE EDUCACIÓN (2010): "Estrategia Universidad 2015". (http://www.educacion.gob.es/dctm/eu2015/2010-pdf eu2015.pdf?documen$\mathrm{tId}=0901 \mathrm{e} 72 \mathrm{~b} 801 \mathrm{ee} 2 \mathrm{a} 4)(10-02-2011)$

OFICINA DE COOPERACIÓN UNIVERSITARIA (2010): "2020 Tendencias Universidad: estudio de prospectiva". (http://web.ocu.es/u2020/inicio/Documentos_Generales/Libro\%20Tendencias\%20Universidad\%202020.pdf) (05-05-2011)

OOSTERBEEK, Hessel; GROOT, Wim \& HARTOG, Joop (1992): “An Empirical Analysis of University Choice and Earnings". The Economist , 140 , 3 , 293-309.

PITMAN, Tim (2000): "Perceptions of academics and students as customers: a survey of administrative staff in higher education". Journal of Higher Education Policy and Management, Vol. 22 , 2, 165-75.

QURESHI, Salim (1995): “College accession research: New variables in an old equation”. Journal of Professional Services Marketing, 12(10), 212.

RAHONA LÓPEZ, Marta (2006): "La influencia del entorno socioeconómico en la realización de estudios universitarios: una aproximación al caso español de la década de los 90". Hacienda Pública Española/ Revista de Economía Pública, 178. (3/2006), 55-80.

SEVIER, Robert A. (1993): "Recruiting African-American Undergraduates: A national survey of the factors that affect institutional Choice". College and University, 68(1), 48-52.

SHUPE, David A. (1999): "Productivity, quality, and accountability in higher education". Journal of Continuing Higher Education, Vol. 47, 1, 2-13. 
SOUTAR, Geoffrey. N. \& TURNER, Julia P. (2002): "Students' preferences for university: A conjoint analysis". International Journal of Educational Management, 16(1), 40-45.

TCHIBOZO, Guy (2007): "Extra-Curricular Activity and the Transition from Higher Education to Work: A Survey of Graduates in the United Kingdom". Higher Education Quarterly, Vol.61, 1,37-56.

TIERNEY, William G. (1999): Building the Responsive Campus: Creating High Performance Colleges and Universities. Thousand Oaks: Sage Publications.

VELOUTSOU, Cleopatra; LEWIS, John W.; \& PATON, Robert A. (2004): "University selection: information requirements and importance. International Journal of Educational Management, 18(3), 160-171. 